\title{
Downregulation of microRNA-21 enhances radiosensitivity in nasopharyngeal carcinoma
}

\author{
HONGHAI ZHU ${ }^{1}$, XIAOYUAN ZHU ${ }^{2}$, GENYANG CHENG $^{3}$, MINGHUI ZHOU ${ }^{1}$ and WEIHUA LOU ${ }^{2}$ \\ Departments of ${ }^{1}$ Rhinology, ${ }^{2}$ Otorhinolaryngology-Head and Neck Surgery and ${ }^{3}$ Nephrology, \\ The First Affiliated Hospital of Zhengzhou University, Zhengzhou, Henan 450052, P.R. China
}

Received July 5, 2014; Accepted March 16, 2015

DOI: $10.3892 /$ etm.2015.2403

\begin{abstract}
Radioresistance severely restricts the clinical treatment of nasopharyngeal carcinoma (NPC). microRNAs (miRs) have been demonstrated to affect cancer progression and radiosensitivity. Thus, the aim of the present study was to identify miRs associated with radioresistance in NPC. A radioresistant NPC cell line (CNE-2-1) was established by continuously exposing CNE-2 cells to radiation. Subsequently, high-throughput sequencing technology was used to detect the regulation of miRs in radioresistant CNE-2-1 cells, and it was observed that miR-21 was among the three most upregulated miRs in CNE-2-1 cells. Therefore, the expression levels of miR-21 were quantified using reverse transcription-quantitative polymerase chain reaction. Finally, the function of miR-21 was investigated by downregulating the expression in the CNE-2-1 cells. The results indicated that the expression of miR-21 was significantly increased in the CNE-2-1 cells, as compared with the CNE-2 cells. In addition, downregulation of miR-21 resulted in enhanced radiosensitivity in the CNE-2-1 cells, as demonstrated by the inhibition in cell viability of these radioresistant cells. Further analysis indicated that miR-21 was able to inhibit the proliferation of CNE-2-1 cells at the G1 phase of the cell cycle. Therefore, these results indicated that miR-21 was able to regulate radioresistance in NPC cells; however, further studies are required to confirm this hypothesis.
\end{abstract}

\section{Introduction}

microRNAs (miRs) are a class of small (19-24 nucleotides), non-coding RNAs that are involved in post-transcriptional gene regulation and/or degradation $(1,2)$. miRs serve a crucial function in the proliferation, differentiation and metabolism

Correspondence to: Mr. Genyang Cheng, Department of Nephrology, The First Affiliated Hospital of Zhengzhou University, 1 Jianshe East Road, Zhengzhou, Henan 450052, P.R. China

E-mail: genyangcheng@126.com

Key words: microRNA-21, radioresistance, nasopharyngeal carcinoma of a wide range of plant and animal cell types $(3,4)$. In order to prevent translation or contribute to target mRNA degradation, miRs bind to target $\mathrm{mRNAs}$ at the 3'-untranslated region (UTR) and/or the 5'-UTR (5).

Nasopharyngeal carcinoma (NPC) is a non-lymphomatous, squamous cell malignancy arising from the epithelial lining of the nasopharynx, and is particularly common in Southeast Asia (6). NPC is an Epstein-Barr virus-associated cancer (7). The incidence rate of NPC is 30-80/100,000 individuals per year, a rate that has remained consistently high for decades (8). The early symptoms of NPC are not often evident and the majority of patients with NPC are not diagnosed until the advanced stages of the disease. Radiotherapy and chemotherapy are the most common treatment options for NPC, and although the radiotherapy technology has improved considerably over the previous decade, the NPC survival rate remains low (9). A key reason for this is the resistance of NPC cells to radiation. Thus, there is a crucial requirement for studies to elucidate the mechanisms underlying NPC radiation resistance.

A previous study by Zhang et al indicated associations between the tumor-related genes, c-Myc, SPLUNC1, Brd3 and UBAP1, with miR-141 in NPC cells (10). Furthermore, Xia et al (11) and Wong et al (12) indicated that the let-7 family of miRs was able to inhibit the proliferation of NPC cells. In addition, Shi et al (13) observed that miR-100 was able to upregulate PLK1 expression, which resulted in the progression of NPC. To date, few studies have investigated the association between radiation resistance and miR-21 in NPC cells.

In the present study, continual radiation was applied to a CNE-2 NPC cell line in order to acquire radioresistant (CNE-2-1) NPC cells. A high-throughput miR sequencing assay was subsequently used to analyze the regulation of miR between the two cell lines. The aim of the present study was to investigate the association between radioresistance and miR-21 in NPC cells.

\section{Materials and methods}

Cell culture. The CNE-2 NPC cell line (Shanghai Biological Technology Co., Ltd., Shanghai, China) was cultured in RPMI 1640 medium (Invitrogen Life Technologies, Carlsbad, CA, USA), supplemented with $10 \%$ fetal bovine serum (Invitrogen Life Technologies), $100 \mathrm{IU} / \mathrm{ml}$ penicillin and 
$100 \mathrm{IU} / \mathrm{ml}$ streptomycin (Invitrogen Life Technologies), at $37^{\circ} \mathrm{C}$ under $5 \% \mathrm{CO}_{2}$ in a humidified incubator.

Establishing a radioresistant NPC CNE-2-1 cell line. CNE-2 cells were cultured in a T75 flask (Corning Incorporated, Corning, New York, NY, USA) and subjected to 2 Gy irradiation (IR) using a RS 2000 biological irradiator (Rad Source Technologies, Inc., Suwanee, GA, USA). Following the first radiation exposure, the cells were cultured and passaged twice. The surviving cells were subsequently treated with the same assay as previously described; however, the IR dose was increased to 4, 6, 8 and 10 Gy after each dose had been administered twice. Following the complete radiation treatment, the surviving cells were cultured and defined as the radioresistant NPC cell line, named CNE-2-1. CNE-2 cells that received no radiation exposure were used as a control cell line.

Cell viability assay. Cell viability was assessed using a Cell Counting Kit (CCK)-8 assay (Beyotime Institue of Biotechnology, Haimen, China), according to the manufacturer's instructions. Briefly, cells that received various treatments (treated with LNA-antimiR-21 or LNA-control) were cultured in triplicate in a 96-well plate for $24 \mathrm{~h}$, after which the cells were subjected to the assigned IR dose. CCK- 8 reagent was added to each well for $2 \mathrm{~h}$ prior to the termination of the experiment. Absorbance values were measured using a VersaMax Microplate Reader (Molecular Devices, LLC, Sunnyvale, CA, USA) and expressed as the viability percentages of the cells compared with the control cells. All experiments were performed in triplicate and the data are presented as the mean \pm standard deviation.

Locked nucleic acid (LNA)-antimiR-21 transfection assay. CNE-2-1 cells were maintained in RPMI 1640 medium. For transfection, the LNA-antimiR-21 or LNA-control oligonucleotides (Exiqon A/S, Vedbaek, Denmark) were administered at a final concentration of $50 \mathrm{nM}$ using Lipofectamine 2000 reagent (Invitrogen Life Technologies).

Cell cycle analysis. Briefly, the CNE-2-1 cells transfected with LNA-antimiR-21 or LNA-control were exposed to 4 Gy IR, cultured for 3 days and then harvested on day 4. After rinsing twice with cold phosphate-buffered saline, the cells were fixed with $70 \%$ paraformaldehyde at $4^{\circ} \mathrm{C}$. Subsequently, the cells were treated with RNase A (Beyotime) for $30 \mathrm{~min}$, followed by treatment with trypsin $(0.5 \% \mathrm{w} / \mathrm{v}$; Beyotime $)$ and ethylenediaminetetraacetic acid $(0.2 \% \mathrm{w} / \mathrm{v})$ for $5 \mathrm{~min}$. Finally, the cells were stained with $50 \mu \mathrm{g} / \mathrm{ml}$ propidium iodide (Beyotime) and analyzed using a BD FACSCalibur flow cytometer (BD Biosciences, Franklin Lakes, NJ, USA). A total of 30,000 events were analyzed for each sample. All tests were performed in triplicate and the data are presented as the mean \pm standard deviation.

Reverse transcription-quantitative polymerase chain reaction (RT-qPCR) assay. Total RNA was extracted from the CNE-2-1 cells transfected with LNA-antimiR-21 or LNA-control using TRIzol reagent (Invitrogen Life Technologies), according to the manufacturer's instructions. For analysis of miR-21 expression, the stem-loop RT primer, qPCR primers and probe were designed as previously described (14). Initially, the miR was reverse transcribed into cDNA using Super-Script II reverse transcriptase (Invitrogen). qPCR was performed using a standard TaqMan PCR protocol and a LightCycler 480 II PCR system (Roche Diagnostics, Basel, Switzerland), according to the manufacturer's instructions. The relative expression levels were calculated using the $2^{-\Delta \Delta \mathrm{Ct}}$ method and were normalized against the expression levels of U6 RNA. All RT-qPCR assays were performed in triplicate and the data are presented as the mean \pm standard deviation.

Statistical analysis. Data are expressed as the mean \pm standard deviation. Statistical analysis was performed with the $t$-test using SPSS statistical software, version 13.0 (SPSS, Inc., Chicago, IL, USA) to evaluate the statistical significance of the differences between groups. $\mathrm{P}<0.05$ was considered to indicate a statistically significant difference.

\section{Results}

Establishment of the radioresistant CNE-2-1 cell line. In order to acquire radioresistant NPC cells, CNE-2 cells were subjected to a series of increasing IR doses. After a total IR dose of $60 \mathrm{~Gy}$, the surviving cells were harvested, cultured and designated as radioresistant $\mathrm{CNE}-2-1$ cells. To analyze the radioresistant capacity of the CNE-2-1 cells, CNE-2-1 and CNE-2 cells were exposed to varying doses of IR, and the cell viability was assessed using a CCK-8 assay. After exposure to 6 Gy IR on day 0 , the two cell lines were cultured for 5 days. Cell viability was assessed every day, and the results indicated that the viability of the CNE-2-1 cells was significantly enhanced compared with the CNE-2 cells. The effect was notable at day 3 following radiation exposure (Fig. 1). In addition, the CNE-2-1 and CNE-2 cell lines were exposed to a range of IR doses $(0,2,4,6$ or 8 Gy) every day, and the cell viability was assessed on day 4 . The results indicated that the radiation exposure reduced the viability of the CNE-2-1 and CNE-2 cells in a dose-dependent manner. Compared with the CNE-2 cells, radiation exposure exhibited less inhibition on the CNE-2-1 cells, and the difference in cell viability at each IR concentration between the CNE-2-1 and CNE-2 cell lines was statistically significant (Fig. 2). Therefore, the CNE-2-1 cells demonstrated a marked radioresistance compared with the CNE-2 cells, and the CNE-2-1 cell model of radioresistant NPC cells was determined to have been successfully established, while untreated CNE-2 cells were used as the control.

Regulation of miR in CNE-2-1 and CNE-2 cells. To investigate the difference in miR expression between the CNE-2-1 and CNE-2 cell lines, cells from each cell line were cultured in a 6-well plate, harvested and subjected to a high-throughput miR sequencing assay. The results indicated that $16 \mathrm{miRs}$ were upregulated, while $33 \mathrm{miRs}$ were downregulated in the CNE-2-1 cell line (data not shown). The altered regulation of specific miRs has been reported to play a role in tumor development, including tumor radiation resistance $(15,16)$. Therefore, it was hypothesized that the regulation of these miRs may contribute to the radioresistance observed in NPC cells. Subsequently, the miRs with altered regulation in the CNE-2-1 cells were selected to determine any association with 


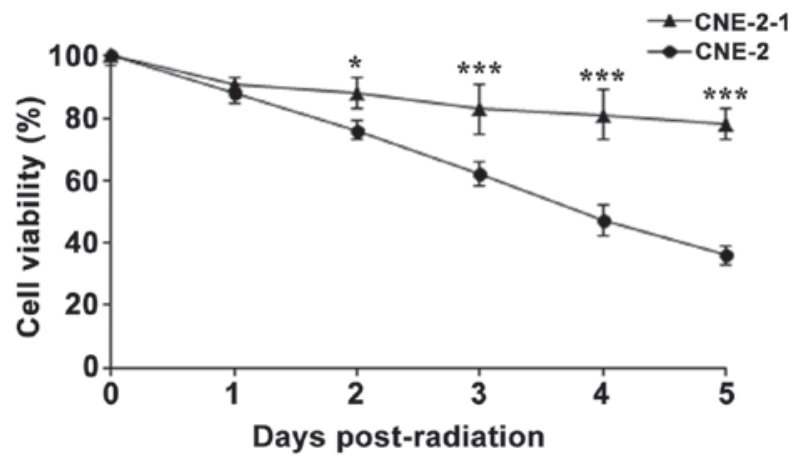

Figure 1. Cell viability of CNE-2-1 and CNE-2 cell lines following irradiation. The cells were exposed to $6 \mathrm{~Gy}$ irradiation on day 0 , and the cell viability was assessed using a Cell Counting Kit-8 assay. Results are expressed as the mean \pm standard deviation of three independent experiments. " $\mathrm{P}<0.05$ and ${ }^{* * * *} \mathrm{P}<0.001$, vs. CNE- 2 .

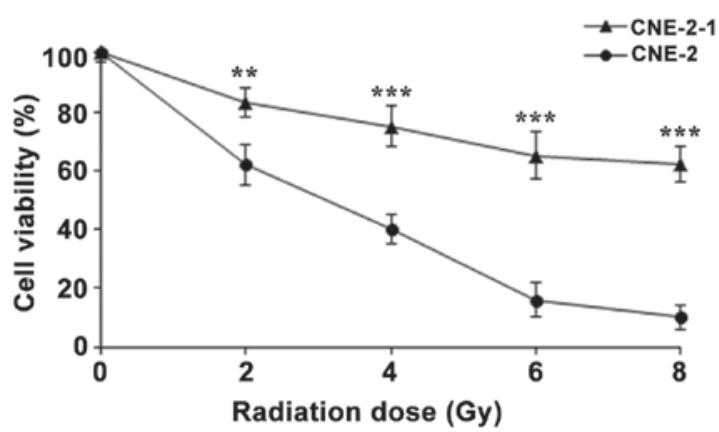

Figure 2. Cell viability of CNE-2-1 and CNE-2 cell lines on day 4 following various irradiation doses. The cells were exposed to a different dose of irradiation $(0,2,4,6$ and $8 \mathrm{~Gy})$ every day and the cell viability was assessed on day 4 using a Cell Counting Kit- 8 assay. Results are expressed as the mean \pm standard deviation of three independent experiments. ${ }^{*} \mathrm{P}<0.05$ and ${ }^{* * *} \mathrm{P}<0.001$, vs. CNE- 2 .

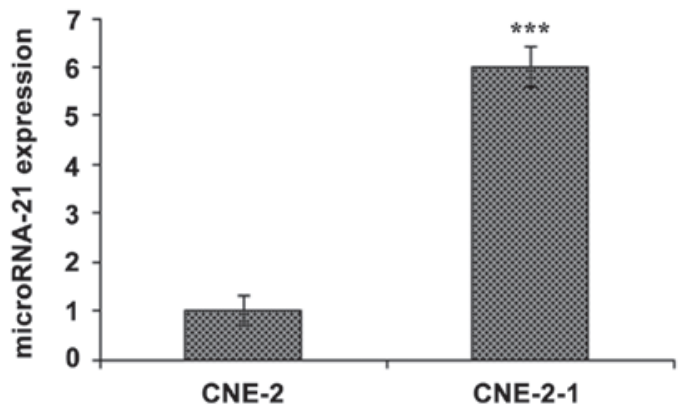

Figure 3. microRNA-21 expression in the CNE-2-1 and CNE-2 cell lines. Relative expression levels were assessed using reverse transcription-quantitative polymerase chain reaction and calculated using the $2^{-\Delta \Delta \mathrm{Ct}}$ method. Expression levels were normalized against the expression of U6 RNA. Results are expressed as the mean \pm standard deviation of three independent experiments. ${ }^{* * *} \mathrm{P}<0.001$, vs. CNE-2.

radioresistance in NPC. miR-21 was among the three most upregulated miRs detected in CNE-2-1 cells and to the best of our knowledge there were no previous studies regarding this area. Therefore, miR-21 was selected for further investigation.

Quantification of miR-21 expression levels using RT-qPCR. To confirm the upregulation of miR-21 in CNE-2-1 cells,

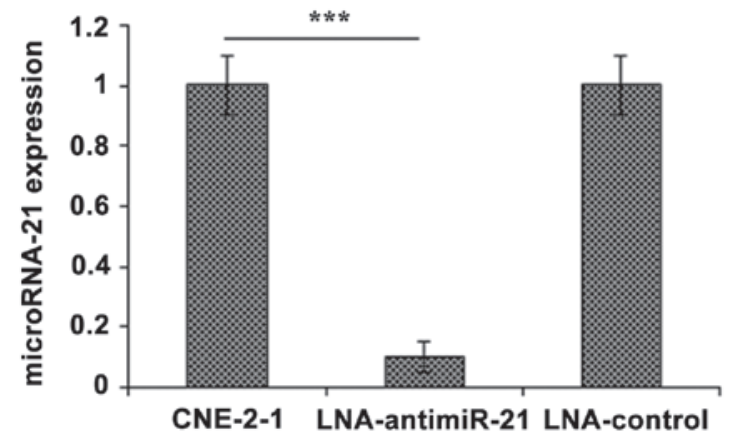

Figure 4. microRNA-21 expression in CNE-2-1 control cells and CNE-2-1 cell lines transfected with LNA-antimicroRNA-21 or LNA-control. Relative expression levels were assessed using reverse transcription-quantitative polymerase chain reaction and calculated using the $2^{-\Delta \Delta \mathrm{Ct}}$ method. Expression levels were normalized against the expression of U6 RNA. Results are expressed as the mean \pm standard deviation of three independent experiments. ${ }^{* * *} \mathrm{P}<0.001$ vs. CNE-2-1. LNA, locked nucleic acid; miR, microRNA.

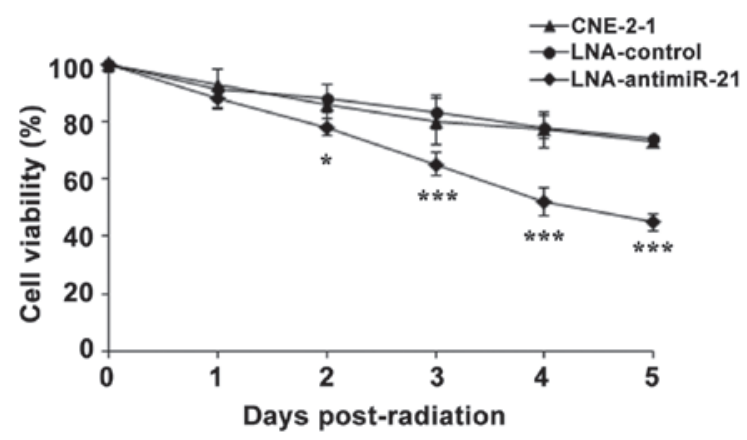

Figure 5. Cell viability of CNE-2-1 control cells and CNE-2-1 cell lines transfected with LNA-antimicroRNA-21 or LNA-control. The cells were exposed to $6 \mathrm{~Gy}$ irradiation on day 0 , and the cell viability was assessed using a Cell Counting Kit- 8 assay. Results are expressed as the mean \pm standard deviation of three independent experiments. ${ }^{*} \mathrm{P}<0.05$ and ${ }^{* * *} \mathrm{P}<0.001$ vs. CNE-2-1. LNA, locked nucleic acid; miR, microRNA.

CNE-2-1 and CNE-2 cells were cultured in a 6-well plate, harvested and subjected to RT-qPCR. The results indicated that miR-21 expression was significantly upregulated ( $\sim 6$ fold) in the CNE-2-1 cells, as compared with the CNE-2 cells (Fig. 3).

In order to determine whether downregulation of miR-21 was able to increase the radiosensitivity of the CNE-2-1 cells, an LNA-antimiR-21 transfection assay was performed. CNE-2-1 cells were transfected with LNA-antimiR-21 or LNA-control oligonucleotides for $48 \mathrm{~h}$, harvested and subjected to RT-qPCR. The results indicated that the expression levels of miR-21 were significantly reduced following transfection with the LNA-antimiR-21 oligonucleotide (Fig. 4). Therefore, downregulation of miR-21 using LNA-antimiR-21 transfection was applied in the further experiments to assess the function of miR-21 in NPC.

Downregulation of miR-21 increases the radiosensitivity of CNE-2-1 cells. In order to investigate whether the upregulation of miR-21 was associated with the radioresistance of CNE-2-1 cells, further cell viability assays were performed. CNE-2-1 control cells, CNE-2-1 cells transfected with LNA-antimiR-21 and LNA-control cells were exposed to $6 \mathrm{~Gy}$ 

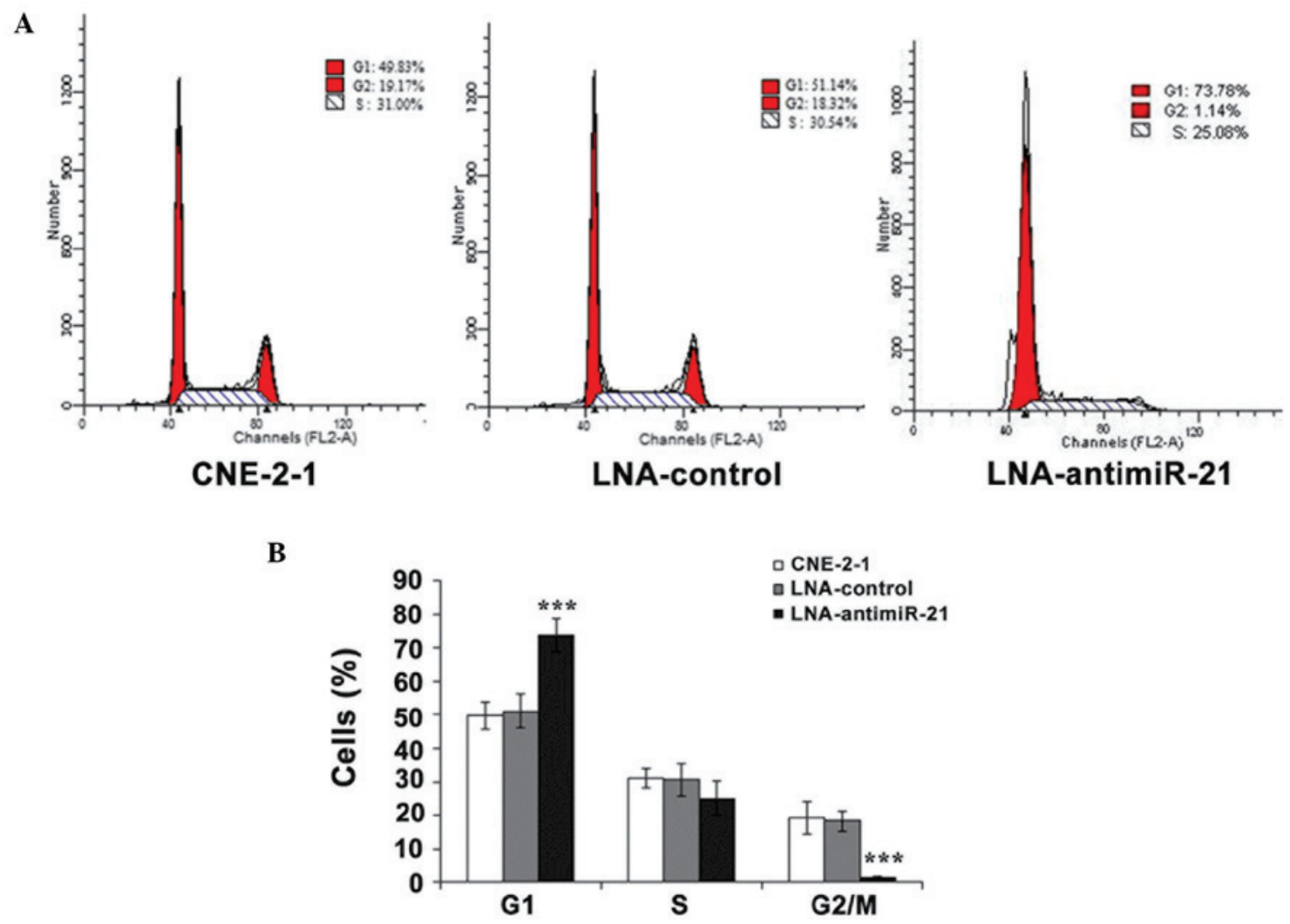

Figure 6. (A and B) Cell cycle distribution in CNE-2-1 control cells and CNE-2-1 cell lines transfected with LNA-antimicroRNA-21 or LNA-control. Results are expressed as the mean \pm standard deviation of three independent experiments. ${ }^{* * * *} \mathrm{P}<0.001$, vs. CNE-2-1. LNA, locked nucleic acid; miR, microRNA.

IR on day 0 and cultured for 5 days. The cell viability was assessed every day using a CCK-8 assay. The results indicated that downregulation of miR-21 significantly inhibited the viability of the radiation-exposed CNE-2-1 cells, as compared with the untreated CNE-2-1 cells, while no difference was observed in the CNE-2-1 control cells compared with the untreated CNE-2-1 cells (Fig. 5). Thus, the downregulation of miR-21 was demonstrated to increase the radiosensitivity of CNE-2-1 cells.

Downregulation of miR-21 affects the cell cycle of CNE-2-1 cells. Cell cycle assays were performed to determine whether the inhibition of CNE-2-1 cell viability was associated with cell cycle regulation. CNE-2-1 control cells and CNE-2-1 cells transfected with LNA-antimiR-21 or LNA-control were exposed to 4 Gy IR for 3 days, after which the cells were harvested and subjected to fluorescence-activated cell sorting (FACS) for cell cycle analysis. The results indicated that the percentage of cells at the G1 phase in the LNA-antimiR-21 group was significantly increased, while the percentage at the G2/M phase was significantly reduced when compared with the CNE-2-1 control cells (Fig. 6). Thus, the results from the FACS assay indicated that downregulation of miR-21 inhibited CNE-2-1 cell proliferation by disrupting the G1 phase of the cell cycle.

\section{Discussion}

NPC is a common disease in Southeast Asia, particularly amongst the Cantonese population of southern China, including those in the Guangdong and Guangxi provinces. Since the majority of patients are diagnosed at an advanced stage of the disease, radiotherapy is the primary therapeutic strategy for patients (17). Although developments in radiotherapy technology have led to improved NPC treatment, the efficacy of radiotherapy remains limited (18). Radioresistant cells are considered to be the primary reason for this limited efficacy; however, the mechanisms underlying NPC cell radioresistance are unclear.

miRs are a class of small, non-coding RNA molecules that function to repress translation or degrade mRNA, subsequently contributing to the inhibition of gene expression $(19,20)$. miRs serve crucial functions in a wide range of physiological and pathological processes, including tumorigenesis (21). Increasing evidence implicates miRs with various processes associated with cancer progression, including tumor growth, differentiation, invasion, metastasis and angiogenesis (22-24). A number of miRs are known to be dysregulated in NPC cell lines (25). For example, miR-125a-5p has been demonstrated to regulate and function as a prognostic factor for gefitinib treatment in NPC (26). Furthermore, upregulation of miR-324-3p has been shown to inhibit radioresistance in NPC cells (27). miR-21 was one of the first miRs to be identified in mammals; and is highly conserved across mammal species. Previous studies have demonstrated that miRs are expressed in numerous types of cancer, including lung, prostate and liver (28). In addition, Deng et al observed that miR-21 was highly expressed in NPC (29). The present study aimed to investigate the association between miR-21 and NPC radioresistance. 
A CNE-2 NPC cell line was continually exposed to radiation in order to obtain a radioresistant NPC cell line. Following the application of a total IR dose of $60 \mathrm{~Gy}$, the surviving CNE-2 cells were considered to have developed a marked radiation resistance. The radioresistant CNE-2-1 cells were subsequently used to investigate the association between miRs and the radioresistance of NPC cells. A high-throughput miR sequencing assay identified 16 upregulated and 33 downregulated $\mathrm{miRs}$, and the expression of $\mathrm{miR}-21$ was observed to be upregulated 6-fold in the CNE-2-1 cells using a RT-qPCR assay. CCK-8 cell viability assays indicated that downregulation of miR-21 significantly enhanced the radiosensitivity of the CNE-2-1 cells. Furthermore, the downregulation of miR-21 was shown to inhibit CNE-2-1 cell proliferation at the G1 phase. However, the molecular mechanism underlying the effects of miR-21 on CNE-2-1 cells remains largely unclear. Therefore, the results of the present study outline the novel regulation of miR-21 in CNE-2 radioresistant cells.

In conclusion, the key finding of the present study is the identification of a potential target of radioresistance in NPC CNE-2 cells. Therefore, improved understanding of the functional interaction between miR-21 and radioresistance in NPC cells may lead to future therapeutic methods.

\section{References}

1. Ambros V: The functions of animal microRNAs. Nature 431: 350-355, 2004

2. Bartel DP: MicroRNAs: Genomics, biogenesis, mechanism and function. Cell 116: 281-297, 2004.

3. He H, Jazdzewski K, Li W, et al: The role of microRNA genes in papillary thyroid carcinoma. Proc Natl Acad Sci USA 102 19075-19080, 2005.

4. Voorhoeve PM, le Sage C, Schrier M, et al: A genetic screen implicates miRNA-372 and miRNA-373 as oncogenes in testicular germ cell tumors. Cell 124: 1169-1181, 2006.

5. Wu L, Fan J and Belasco JG: MicroRNAs direct rapid deadenylation of mRNA. Proc Natl Acad Sci USA 103: 4034-4039, 2006.

6. Wei WI and Sham JS: Nasopharyngeal carcinoma. Lancet 365 : 2041-2054, 2005.

7. Lin J-C: Adjuvant chemotherapy in advanced nasopharyngeal carcinoma based on plasma EBV load. J Radiat Oncol 1: 117-127, 2012.

8. Li T, Chen JX, Fu XP, et al: microRNA expression profiling of nasopharyngeal carcinoma. Oncol Rep 25: 1353-1363, 2011.

9. Jemal A, Siegel R, Ward E, et al: Cancer statistics, 2009. CA Cancer J Clin 59: 225-249, 2009.

10. Zhang L, Deng T, Li X, et al: microRNA-141 is involved in a nasopharyngeal carcinoma-related genes network. Carcinogenesis 31 : $559-566,2010$
11. Xia H, Ng SS, Jiang S, et al: miR-200a-mediated downregulation of ZEB2 and CTNNB1 differentially inhibits nasopharyngeal carcinoma cell growth, migration and invasion. Biochem Biophys Res Commun 391: 535-541, 2010.

12. Wong TS, Man OY, Tsang CM, et al: MicroRNA let-7 suppresses nasopharyngeal carcinoma cells proliferation through downregulating c-Myc expression. J Cancer Res Clin Oncol 137: 415-422, 2011.

13. Shi W, Alajez NM, Bastianutto C, et al: Significance of Plk1 regulation by miR-100 in human nasopharyngeal cancer. Int J Cancer 126: 2036-2048, 2010.

14. Chen C, Ridzon DA, Broomer AJ, et al: Real-time quantification of microRNAs by stem-loop RT-PCR. Nucleic Acids Res 33: e179, 2005.

15. Calin GA, Sevignani C, Dumitru CD, et al: Human microRNA genes are frequently located at fragile sites and genomic regions involved in cancers. Proc Natl Acad Sci USA 101: 2999-3004, 2004.

16. Sevignani C, Calin GA, Nnadi SC, et al: MicroRNA genes are frequently located near mouse cancer susceptibility loci. Proc Natl Acad Sci USA 104: 8017-8022, 2007.

17. Fåhraeus R, Fu HL, Ernberg I, et al: Expression of Epstein-Barr virus-encoded proteins in nasopharyngeal carcinoma. Int J Cancer 42: 329-338, 1988.

18. Jemal A, Siegel R, Xu J and Ward E: Cancer statistics, 2010. CA Cancer J Clin 60: 277-300, 2010.

19. Sun K and Lai EC: Adult-specific functions of animal microRNAs. Nat Rev Genet 14: 535-548, 2013.

20. Ameres SL and Zamore PD: Diversifying microRNA sequence and function. Nat Rev Mol Cell Biol 14: 475-488, 2013.

21. van Kouwenhove M, Kedde M and Agami R: MicroRNA regulation by RNA-binding proteins and its implications for cancer. Nat Rev Cancer 11: 644-656, 2011.

22. Kasinski AL and Slack FJ: Epigenetics and genetics. MicroRNAs en route to the clinic: Progress in validating and targeting microRNAs for cancer therapy. Nat Rev Cancer 11: 849-864, 2011.

23. Iorio MV and Croce CM: MicroRNA dysregulation in cancer: Diagnostics, monitoring and therapeutics. A comprehensive review. EMBO Mol Med 4: 143-159, 2012.

24. Valencia-Sanchez MA, Liu J, Hannon GJ and Parker R: Control of translation and mRNA degradation by miRNAs and siRNAs. Gene Dev 20: 515-524, 2006

25. Chen HC, Chen GH, Chen YH, et al: MicroRNA deregulation and pathway alterations in nasopharyngeal carcinoma. $\mathrm{Br}$ J Cancer 100: 1002-1011, 2009.

26. Liu Y, Li Z, Wu L, et al: MiRNA-125a-5p: A regulator and predictor of gefitinib's effect on nasopharyngeal carcinoma. Cancer Cell Int 14: 24, 2014.

27. Li G, Liu Y, Su Z, et al: MicroRNA-324-3p regulates nasopharyngeal carcinoma radioresistance by directly targeting WNT2B. Eur J Cancer 49: 2596-2607, 2013.

28. Lu J, Getz G, Miska EA, et al: MicroRNA expression profiles classify human cancers. Nature 435: 834-838, 2005.

29. Deng M, Gu Y, Zheng G, et al: Expression and clinical significance of miR-21 in nasopharyngeal carcinoma. Shandong Med J 52: 10-12, 2012. 